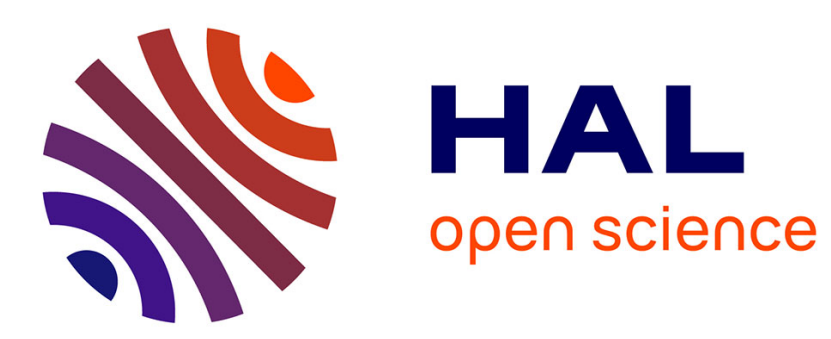

\title{
Dynamic Sleep Mode for Minimizing a Femtocell Power Consumption
}

\author{
Rémi Bonnefoi, Christophe Moy, Jacques Palicot
}

\section{To cite this version:}

Rémi Bonnefoi, Christophe Moy, Jacques Palicot. Dynamic Sleep Mode for Minimizing a Femtocell Power Consumption. 11th International Conference on Cognitive Radio Oriented Wireless Networks, CROWNCOM 2016, May 2016, Grenoble, France. pp.618-629, 10.1007/978-3-319-40352-6_51 . hal01418838v2

\section{HAL Id: hal-01418838 \\ https://hal.science/hal-01418838v2}

Submitted on 30 Oct 2018

HAL is a multi-disciplinary open access archive for the deposit and dissemination of scientific research documents, whether they are published or not. The documents may come from teaching and research institutions in France or abroad, or from public or private research centers.
L'archive ouverte pluridisciplinaire HAL, est destinée au dépôt et à la diffusion de documents scientifiques de niveau recherche, publiés ou non, émanant des établissements d'enseignement et de recherche français ou étrangers, des laboratoires publics ou privés. 


\title{
Dynamic sleep mode for minimizing a femtocell power consumption
}

\author{
Rémi Bonnefoi ${ }^{1}$, Christophe Moy ${ }^{1}$ and Jacques Palicot ${ }^{1}$ \\ CentraleSupélec/IETR, CentraleSupélec Campus de Rennes, 35510 Cesson-Sévigné, \\ France, \\ \{remi.bonnefoi, christophe.moy, jacques.palicot\}@centralesupelec.fr
}

\begin{abstract}
The use of power control (PC) and discontinuous transmission (DTx) can reduce the average power consumption of mobile base stations (BS). In this paper, the power consumption of a picocell or a femtocell are analyzed in a time division multiple access (TDMA) scenario. The minimization of the power consumption is viewed as a constrained optimization problem and a closed form of the compromise between transmit power and transmission time which minimizes the average power consumption is found. Moreover, we show that for a base station with a low transmit power and a sufficient power saving during sleep mode, such as picocells and femtocells, the average power consumption is minimum when the service time is minimized. Finally, numerical results show that discontinuous transmission greatly decreases the average power consumption of the base station.
\end{abstract}

Key words: Sleep mode, Discontinuous transmission, green communications

\section{Introduction}

Decreasing the power consumption of a mobile network allows to reduce both the operating cost for mobile operator and the ecological footprint of the network. It has been shown that deploying more and more pico and femto base stations can reduce the global carbon footprint of mobile communication technologies [1]. Moreover, power control and cell DTx allow to curtail power consumption without reducing users quality of service (QoS).

With cell DTx, the base station can switch between active and sleep mode to reduce the average power consumption. Two kinds of DTx are possible, dynamic and quasi-static DTx [2]. With quasi-static DTx cells are switched off or into deep sleep mode during off peak hours (for some minutes or hours) if there is no users in the cell coverage. Quasi-static DTx is often used with cell shaping to reduce the energy consumption of wireless networks [3]. On the contrary, with dynamic DTx, the base station is switched to sleep mode for a very short time (a few microseconds). During those short sleeping periods, some of the most consuming elements of the radio frequency chain such as the power amplifier are switched off. These two type of DTx were studies during the EARTH project $[4]$. 
An example of use of dynamic DTx can be found in the LTE standard. Indeed, in this standard, a $10 \mathrm{~ms}$ frame is composed by ten $1 \mathrm{~ms}$ sub-frames and up to six of those sub-frames can be MBSFN (multicast broadcast single frequency network). During a MBSFN sub-frame, the base station is switched to sleep mode and the power consumption reduced [5]. In [6], the reduction of power consumption induced by MBSFN sub-frames is studied. In [7], the power amplifier is switched on and off to save energy while maintaining the cell coverage and quality of service. In those three papers, power control is not used and user are always served using the whole available power. Contrary to papers previously mentioned which only use DTx, in this paper we analyze the joint use of PC and dynamic DTx to save energy.

In this paper, we suppose that a picocell or a femtocell serves several users and knows the channel gain and the throughput demand (capacity demand) of each of them. Time is divided in short time frames (which, for example, can last $1,5,10$ or $100 \mathrm{~ms}$ as we do not restrict to LTE context). In each frame, users are served successively using time division multiple access (TDMA) over the whole available band and when all users have been served, the base station switches to sleep mode for the rest of the frame. During each frame, each user is served during a specfic service time (duration of data transmission to this user). This service time must be adjusted so that each user has a certain QoS (capacity demand) while minimizing the average power consumption of the base station. The optimization problem solved in this paper has already been raised in [8] with simulation results but we propose here a closed form solution.

We are in a Cognitive Green Radio (GR) context [9] and the system studies can be modeled as a simplified cognitive cycle in three steps (sensing, decision making and adaptation). During the sensing period, the base station collects the propagation conditions and the capacity constraint of all users. Then, the service time and transmit power are computed during the decision making period and applied during the adaptation period to reduce the power consumption. The simplified cognitive cycle is shown in figure 1 . In this paper we focus on the decision making algorithm.

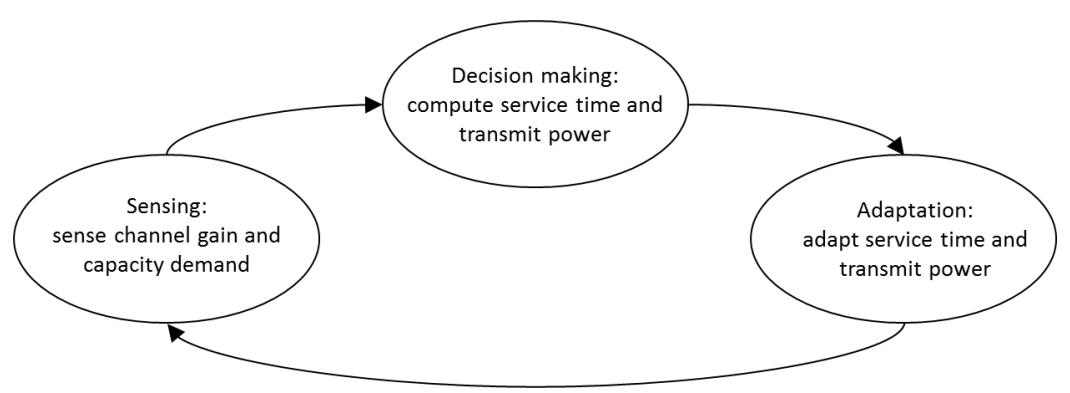

Fig. 1. Simplified cognitive cycle for considered Cognitive Green Radio scenario 
In this paper, the problem of power minimization is first reformulated before proposing a resolution scheme. Then, this problem is solved in a closed form. We show that, in some cases, this closed form solution can be expressed using the W-Lambert function [10] which is the reciprocal bijection of $x \mapsto x e^{x}$. Finally, we show that in a realistic context, the average power consumption of a picocell or a femtocell is minimized by minimizing the service time.

The rest of this paper is organized as follows. In Section II the system model is presented and the optimization problem is introduced. The optimization problem is reformulated in section III and solved in section IV. Finally, in section V numerical simulations illustrate the reduction of power consumption.

\section{System Model}

The power consumption of a base station can be accurately modeled by a linear model [8] :

$$
\left\{\begin{array}{lll}
P_{\text {supply }}= & P_{0}+m P_{T_{X}} \text { if } & 0<P_{T_{X}} \leq P_{\max } \\
P_{\text {supply }}= & P_{s} & \text { else }
\end{array}\right.
$$

Where $P_{0}$ is the static power consumption, $P_{s}$ the power consumed when the base station is in sleep mode. $P_{T_{X}}$ is the transmit power which is below the maximum transmit power of the base station denoted $P_{\text {max }} . m$ is the slope of the load dependence. Figure 2 illustrates the power consumption of the base station during a TDMA frame.

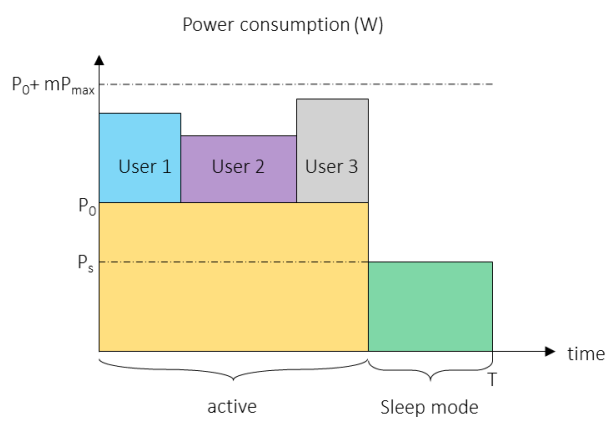

Fig. 2. Variation of the power consumption of a base station during one frame, in this scheme, three users are served by the base station and the base station switches to sleep mode until the end of the frame after having served all

In [11] the authors list some realistic values for $P_{0}, P_{s}, P_{\max }$, and $m$. Those values are mentioned in table 1

In this paper, we denote $N_{u}$ the number of users served by the base station during a frame, $T$ the frame duration and $t_{i}$ the time used to serve user $\mathrm{i}$. 


\begin{tabular}{|c|c|c|c|c|}
\hline BS type & $P_{\max }$ & $P_{0}$ & $m$ & $P_{s}$ \\
\hline Macro & 20.0 & 130.0 & 4.7 & 75.0 \\
\hline Micro & 6.3 & 56.0 & 2.6 & 39.0 \\
\hline Pico & 0.13 & 6.8 & 4.0 & 4.3 \\
\hline Femto & 0.05 & 4.8 & 8.0 & 2.9 \\
\hline
\end{tabular}

Table 1. Power values for different base stations types from [11]

We denote $\mu_{i}$ the proportion of time used to serve user $i$ :

$$
\mu_{i}=\frac{t_{i}}{T}
$$

In a TDMA scheme, all users have the same bandwidth denoted $B$. Moreover, for each user, the expression of the channel capacity is:

$$
C_{i}=B \mu_{i} \log _{2}\left(1+\rho_{i}\right)
$$

Where $\rho_{i}$ is the Signal to Noise Ratio (SNR) of this user. This last is expressed as:

$$
\rho_{i}=\frac{P_{T_{X}}^{i}\left|h_{i}\right|^{2}}{N}=\frac{P_{T_{X}}^{i}\left|h_{i}\right|^{2}}{k T B}=\frac{P_{T_{X}}^{i} G_{i}}{B}
$$

Where $P_{T_{X}}^{i}$ the transmit power used for user $i, h_{i}$ is the channel gain, $N$ denotes the noise power, $k$ is the Boltzmann constant, $T$ the temperature in Kelvin and $G_{i}=\frac{\left|h_{i}\right|^{2}}{k T}$ is a gain proportional to the channel gain of user $i$. For a given value of $\mu_{i}$, there is only one value of the transmit power which allows to exactly meet the capacity constraint $C_{i}$ and its expression is:

$$
P_{T_{X}}^{i}=\frac{B}{G_{i}}\left(2^{\frac{C_{i}}{B \mu_{i}}}-1\right)
$$

Using equations 1 and 5 we can derive the mean power consumption of the base station during one frame:

$$
P_{\text {mean }}=\left(1-\sum_{i=1}^{N_{u}} \mu_{i}\right) P_{s}+\sum_{i=1}^{N_{u}} \mu_{i}\left(P_{0}+m \frac{B}{G_{i}}\left(2^{\frac{C_{i}}{B \mu_{i}}}-1\right)\right)
$$

The objective is to minimize $P_{\text {mean }}$ under constraints:

$$
\begin{gathered}
P_{T_{X}}^{i} \leq P_{\max } \quad \forall i \in \llbracket 1 ; N_{u} \rrbracket \\
\mu_{i} \geq 0 \quad \forall i \in \llbracket 1 ; N_{u} \rrbracket
\end{gathered}
$$

and

$$
\sum_{i=1}^{N_{u}} \mu_{i} \leq 1
$$




\section{Problem reformulation}

\subsection{Existence of solutions}

Before minimizing the average power consumption, we have to analyze the existence of solutions. This problem has a solution if and only if the sum of minimum portions of time required to serve each user is smaller than 1 . We denote $\mu_{i \text { min }}$ the shortest portion of time which ensures the required QoS of user i. The expression of $\mu_{i \min }$ is:

$$
\mu_{i \min }=\frac{C_{i}}{B \log _{2}\left(1+\frac{P_{\max } G_{i}}{B}\right)}
$$

If $\mu_{i}<\mu_{i m i n}$ the transmit power $P_{T_{X}}^{i}$ computed with 5 will be higher than $P_{\max }$ and thus the constraint of equation 7 will not be met. This value is computed by using the maximum transmit power $P_{\max }$ for user i. Thus, the smallest portion of time necessary to serve all users is:

$$
\sum_{i=1}^{N_{u}} \mu_{i \min }=\sum_{i=1}^{N_{u}} \frac{C_{i}}{B \log _{2}\left(1+\frac{P_{\max } G_{i}}{B}\right)}
$$

And, the ability to serve users can be written as $\sum_{i=1}^{N_{u}} \mu_{i \text { min }} \leq 1$, i.e.:

$$
\sum_{i=1}^{N_{u}} \frac{C_{i}}{B \log _{2}\left(1+\frac{P_{\max } G_{i}}{B}\right)} \leq 1
$$

\subsection{New formulation of the optimization problem}

To better understand the problem, we can rewrite equation 6 as:

$P_{\text {mean }}=\underbrace{\left(\sum_{i=1}^{N_{u}} \mu_{i}\right) P_{0}+\left(1-\sum_{i=1}^{N_{u}} \mu_{i}\right) P_{s}}_{\text {Average static power consumption } P_{m}^{\text {stat }}}+\underbrace{m B \sum_{i=1}^{N_{u}} \mu_{i} \frac{1}{G_{i}}\left(2^{\frac{C_{i}}{B \mu_{i}}}-1\right)}_{\text {Average dynamic power consumption } P_{m}^{\text {dyn }}}$

The average static power consumption $P_{m}^{s t a t}$ is minimized when $\sum_{i=1}^{N_{u}} \mu_{i}$ is minimum (when the values of $\mu_{i}$ are set to $\mu_{i \text { min }}$ ). In opposite, the dynamic power consumption $P_{m}^{d y n}$ is minimized when the power is spread over time. Resolving this optimization problem is equivalent to find, for each user, the best compromise between these extremes.

$P_{m}^{s t a t}$ and $P_{m}^{d y n}$ have an upper and a lower bound.

$$
0 \leq P_{m}^{d y n} \leq m P_{\max } \quad \text { and } \quad P_{s} \leq P_{m}^{\text {stat }} \leq P_{0}
$$

Those bounds can be used to see the relative importance of $P_{m}^{s t a t}$ and $P_{m}^{d y n}$. We can note that for femtocells and picocells, $m P_{\max } \ll P_{s}$. Consequently, the 
average static power consumption is much higher than the average dynamic power consumption.

Constraints of equation 7 and 8 can be rewritten in a single constraint on $\mu_{i}$ using $\mu_{i \text { min }}$. These constraints are equivalent to:

$$
\mu_{i} \geq \mu_{i \min } \quad \forall i \in \llbracket 1 ; N_{u} \rrbracket
$$

The last constraint remains unchanged as in equation 9 .

\section{Minimizing power consumption}

In this section, we denote $\mu_{i o p t}$ the value of $\mu_{i}$ which minimizes the power consumption.

\section{1 resolution scheme}

We have,

$$
\frac{\partial^{2} P_{\text {mean }}}{\partial \mu_{i} \partial \mu_{j}}=0 \quad \forall i \neq j
$$

And,

$$
\left.\left.\frac{\partial^{2} P_{\text {mean }}}{\partial^{2} \mu_{i}}=\frac{m B}{G_{i}} 2^{\frac{C_{i}}{B \mu_{i}}}\left(\frac{C_{i}}{B}\right)^{2} \frac{\ln (2)^{2}}{\mu_{i}^{3}} \geq 0 \quad \mu_{i} \in\right] \mu_{i \text { min }} ; 1\right]
$$

Thus, the Hessian matrix of the function of equation 13 is diagonal and the $i^{t h}$ diagonal element of this matrix is positive. As a consequence, the function studied is convex. To solve this non-linear constrained optimization problem with inequality constraints in a closed form, we use the Karush-Kunh-Tucker (KKT) conditions. According to these conditions, each inequality constraint must be considered either as an inequality or as an equality. In other words, among the $2^{N_{u}+1}$ possible Lagrangian, only one leads to the solution. In order to find this Lagrangian and to solve the optimization problem, the following methodology is employed:

- Find the optimum of the problem without constraints. After this resolution, if the result of this calculation satisfies equations 9 and 15 , this solution is optimal and all constraints are strict inequalities constraints;

- If, for user $i$, the value of $\mu_{i \text { opt }}$ found in the preceding step does not satisfies equation 15 , we must set $\mu_{\text {iopt }}=\mu_{\text {imin }}$;

- If those new values of $\mu_{\text {iopt }}$, verify the last constraint (equation 9) then the problem is solved, else, the average power consumption is optimal without employing DTx and is a problem of power allocation. This power allocation problem has already been formulated in [12] but not solved. 


\subsection{Users considered independently}

The values of $\mu_{i}$ which potentially minimize the mean power consumption are:

$$
\mu_{\text {iopt }}=\frac{C_{i} \ln (2)}{B} \frac{1}{\mathcal{W}\left(e^{-1}\left[\frac{G_{i}}{B} \frac{P_{0}-P_{s}}{m}-1\right]\right)+1}
$$

In this equation, the function $\mathcal{W}($.$) is the \mathrm{W}$-Lambert function. This expression of $\mu_{i o p t}$ is always positive and $\mu_{i \text { opt }}$ decreases when $G_{i}$ increases.

If $\mu_{i o p t} \leq \mu_{i \text { min }}, \mu_{i o p t}$ must be set to $\mu_{i \text { min }}$. For each user, the value of $P_{0}-P_{s}$ from which $\mu_{i o p t} \leq \mu_{i \text { min }}$ is :

$$
P_{0}-P_{s}=\left[\frac{m B}{G_{i}}+m P_{\max }\right] \ln \left(1+\frac{P_{\max } G_{i}}{B}\right)-m P_{\max }
$$

Using the inequality:

$$
\ln (1+x) \geq \frac{x}{1+x} \quad \forall x \in \mathbb{R}^{+}
$$

We prove that this value is positive. As a consequence, whatever the value of the $G_{i}$, there is a value of $P_{0}-P_{s}$ from which it is preferable to serve the user with $P_{\max }$ during $\mu_{i \min }$. Moreover, the derivative of this expression with respect to $G_{i}$ is:

$$
\frac{\partial\left(P_{0}-P_{s}\right)}{\partial G_{i}}=\frac{m}{G_{i}}\left[P_{\max }-\frac{B}{G_{i}} \ln \left(1+\frac{P_{\max } G_{i}}{B}\right)\right]
$$

Using the inequality $\ln (1+x) \leq x, \forall x \in \mathbb{R}^{+}$, we can see that this derivative is positive. Consequently, the value of $P_{0}-P_{s}$ from which $\mu_{i \text { opt }}$ is equal to $\mu_{i \text { min }}$ increases with $G_{i}$. Thus, the lower is $G_{i}$, the more we should serve the user during $\mu_{i \text { min }}$.

Moreover, there is a value of $P_{0}-P_{s}$ from which the mean power consumption is minimum when all users are served during $\mu_{i \text { min }}$. To compute this value we assume that the SNR is bounded and we denote $\rho_{\max }$ its maximum. As a consequence, $G_{i}$ can be upperbounded by :

$$
G_{i} \leq \frac{\rho_{\max } B}{P_{\max }}
$$

We denote $\left(P_{0}-P_{s}\right)_{l}$ the value of $P_{0}-P_{s}$ from which all users must be served using $\mu_{i \text { min }}$. This quantity can be computed using equations 19 and 22:

$$
\begin{aligned}
\left(P_{0}-P_{s}\right)_{l} & =m P_{\max }\left(\left(1+\frac{1}{\rho_{\max }}\right) \ln \left(1+\rho_{\max }\right)-1\right) \\
& \approx m P_{\max }\left(\ln \left(\rho_{\max }\right)-1\right)
\end{aligned}
$$

In a cellular network, the SNR is generally below $\rho_{\max }=1000(30 \mathrm{~dB})$. Table 2 lists the value of $\left(P_{0}-P_{s}\right)_{l}$ for each base station type. 


\begin{tabular}{|c|c|c|}
\hline BS type & $\left(P_{0}-P_{s}\right)_{l}$ & Realistic value of $P_{0}-P_{s}$ \\
\hline Macro & 556.1 & 55 \\
\hline Micro & 96.9 & 17 \\
\hline Pico & 3.1 & 2.5 \\
\hline Femto & 2.4 & 1.9 \\
\hline
\end{tabular}

Table 2. Comparison between current and limit value of $P_{0}-P_{s}$

For pico and femto base stations, $\left(P_{0}-P_{s}\right)_{l}$ is slightly higher than current values. Consequently, almost all users will be served during $\mu_{i \text { min }}$ and only a few users will be served during a longer time. Thus, the average power consumption is very close to its minimum when all users are served during $\mu_{i \text { min }}$. In other word, a very simple and efficient way to serve all users optimally is to serve them during $\mu_{i \text { min }}$ with all the available transmit power.

On the contrary, for macro and micro base stations, $\left(P_{0}-P_{s}\right)_{l}$ is greater than $P_{0}$ and much greater than the current value of $P_{0}-P_{s}$.

\subsection{Constraint on the sum}

A consequence of the results of the previous section is that constraint of equation 9 will almost always be met for picocells and femtocells. Moreover, if all users are served during $\mu_{i \text { min }}$ as previously envisaged the constraint on the sum is always met.

For macro and micro base stations, $\left(P_{0}-P_{s}\right)_{l}$ is greater than $P_{0}$ and much greater than the current value of $P_{0}-P_{s}$. In this case, the constraint of equation 9 will not always be met, and more attention should be paid to the management of this case. This case will be studied in a future paper.

\section{Numerical results}

The model proposed in this paper is new and thus it is difficult to compare it with the litterature. We use simulations to compare three different strategies:

- A simple strategy with MBSFN suframes: for each user, the number of subframes is chosen so that the channel capacity exceeds its capacity demand. Only one user can be served in each subframe and up to six subframes can be switched to sleep mode when they are not used to serve one user.

- The use of DTx only, all users are served during $\mu_{i \min }$ with $P_{\max }$.

- The optimal strategy, all users are served during $\mu_{i \text { opt }}$ with the corresponding transmit power.

The base station is a femtocell and its power characteristics are those of table 1 . The number of users served by the base station is defined by a Poisson process with parameter $\lambda=2$. The distance between users and the base station is uniformly distributed between 10 and 200 meters. TDMA is used to manage 
multiple users access. Central frequency is $f=2 G H z$ and a $10 \mathrm{MHz}$ band is used for all users. The pathloss is computed using the ITU pathloss model for indoor propagation [13]. With this model, the expression of the pathloss in a small area is:

$$
L=20 \log _{10}(f)+33 \log _{10}(d)-28+P_{f}\left(n_{\text {floor }}\right)
$$

Where $f$ is the central frequency in $\mathrm{MHz}, \mathrm{d}$ the distance between the user and the base station and $P_{f}\left(n_{\text {floor }}\right)$ the floor penetration loss factor:

$$
P_{f}\left(n_{\text {floor }}\right)=15+4\left(n_{\text {floor }}-1\right) \quad n_{\text {floor }}>0
$$

Where $n_{\text {floor }}$ is the number of floors between the base station and the user, this number is equidistributed between 0 and 5 . A log-normal shadowing with a standard deviation of $10 \mathrm{~dB}$ is added. Moreover, the antenna gain is zero and the noise figure is $9 \mathrm{~dB}$ for all users. We suppose that the SNR is below $\rho_{\max }=30 \mathrm{~dB}$ and that all users have the same capacity constraint.

To compute the minimum power consumption, the following algorithm is used:

1: Compute $\mu_{i \min }$

2: Compute $\mu_{\text {i opt }}$, the optimum without constraint using equation 18

3: if $\mu_{i o p t} \leq \mu_{i \min }$ then

4: $\quad \mu_{\text {iopt }}=\mu_{\text {imin }}$

5: end if

6: if $\sum_{i=1}^{N_{u}}>1$ then

7: $\quad$ use an iterative algorithm to compute the optimum (for example the interior point method)

8: end if

Figure 3 shows the evolution of the average power consumption as a function of the capacity constraint.

If DTx and PC were not used, the average power consumption would be $P_{0}+m P_{\max }=5.2 \mathrm{~W}$. Thus, with MBSFN subframes, the power reduction is between 20 and $26 \%$ whereas, with the proposed DTx, this reduction is between 31 and $42 \%$.

Moreover, we can see that on figure 3 that the circles are on the dashed line. Thus, for picocells and femtocells, it is optimal to use DTx only. Two reasons explain this result:

- For femtocells, the average dynamic power consumption is much lower than the average static power consumption;

- The value of $P_{0}-P_{s}$ is close to the limit value $\left(P_{0}-P_{s}\right)_{l}$ from which all users must be served during $\mu_{i \text { min }}$.

This two reasons can be studied independently of one another. We will first analyze the effect of the reduction of $P_{0}-P_{s}$ before showing the effect of an increase in $P_{\max }$ (increase of the dynamic power consumption). 


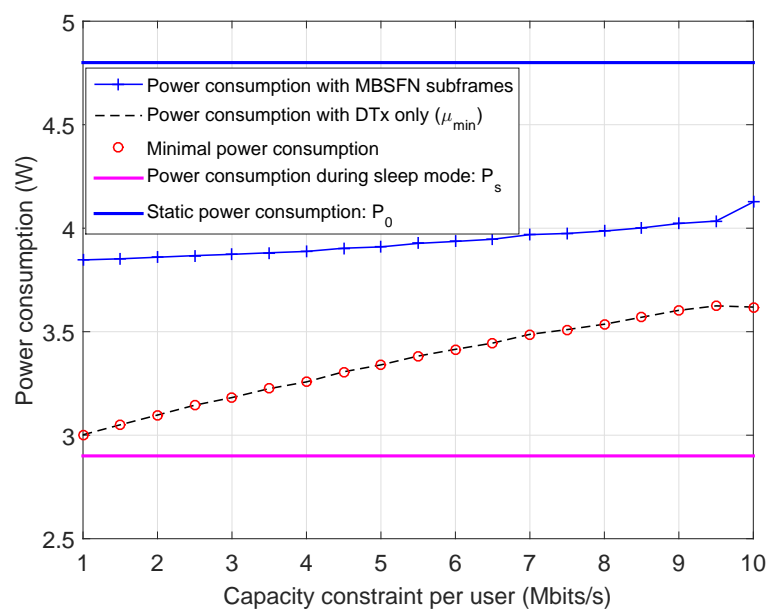

Fig. 3. Comparison between MBSFN subframes, DTx only and the joint use of DTx and power control

On figure 3, the evolution of the mean power consumption is nearly linear. To understand this, we can write the expression of the expectation numerically computed. If all users are served during $\mu_{i \text { min }}$ the expression of this expectation is:

$$
\mathbb{E}\left(P_{\text {mean }}\right)=P_{s}+C \frac{\left[\left(P_{0}-P_{s}\right)+m P_{\text {max }}\right]}{B} \times \mathbb{E}\left(\sum_{i=1}^{N_{u}} \frac{1}{\log _{2}\left(1+\frac{P_{\max } G_{i}}{B}\right)}\right)
$$

Where $\mathrm{C}$ is the capacity constraint. As long as this capacity constraint is not too high, its increase doesn't change the expectation of the total service time.Thus, the evolution is linear. For higher capacity constraint, an increase in capacity changes the number of users served and thus the shape of the curve.

Figure 4 shows the evolution of the mean power consumption versus $P_{s}$.

For this simulation, all propagation conditions and the characteristics of the base station but $P_{s}$ remains unchanged. The increase of $P_{s}$ implies a decrease of $P_{0}-P_{s}$ from $1.2 \mathrm{~W}$ to $0 \mathrm{~W}$.

We can see on figure 4 that when $P_{0}-P_{s}$ decreases, the mean power obtained by serving all users during $\mu_{i \text { min }}$ becomes slightly higher than the theoretical limit.

Figure 5 shows the evolution of the mean power consumption versus $P_{\max }$ for different values of $\mathrm{C}$.

On figure 5 the mean power consumption obtained using $\mu_{i \text { min }}$ is compared with the theoretical minimal power consumption. When $P_{\max }$ increases, the gap between the two curves increases. For example, for a capacity demand of 10 $\mathrm{Mbit} / \mathrm{s}$, the use of $\mu_{i \text { min }}$ instead of $\mu_{i \text { opt }}$ increases the power consumption by $5 \%$ when $P_{\max }=0.25 \mathrm{~W}$ and by $15 \%$ when $P_{\max }=0.5 \mathrm{~W}$. Thus, if $P_{\max }$ is 


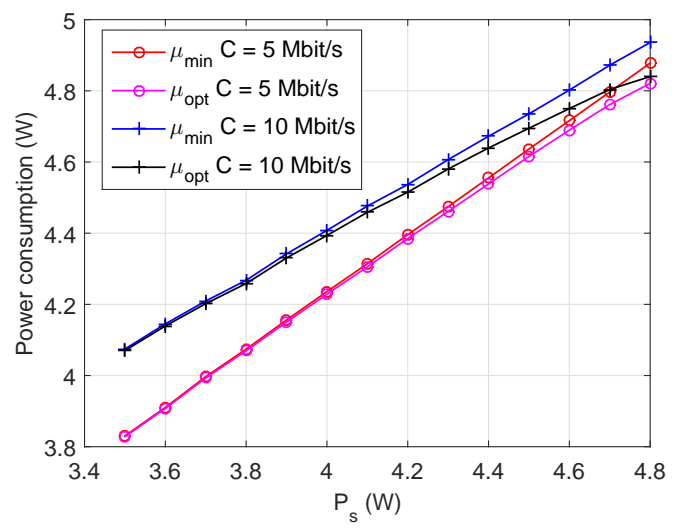

Fig. 4. Evolution of $P_{\text {mean }}$ versus $P_{s}$, an increase in $P_{s}$ slightly influes on the gap between the power consumption with DTx only and the minimal power consumption

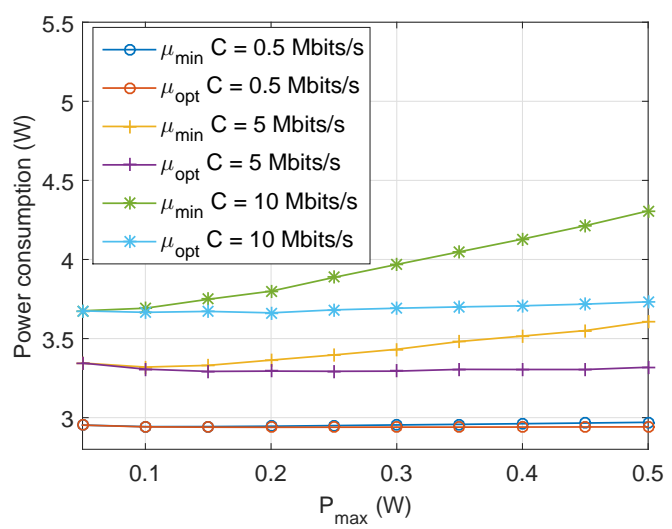

Fig. 5. Evolution of $P_{\text {mean }}$ versus $P_{\text {max }}$, an increase in $P_{\text {max }}$ greatly increases the gap between the power consumption with DTx only and the minimal power consumption

higher than the values indicate in table 1 , it is no longer optimal to use $\mu_{i \min }$ for all users.

\section{Conclusion}

In this paper we studied the compromise between power and time which minimize the average power consumption of a base station in TDMA. We saw that for current picocells and femtocells, the power is minimized when the service time is minimized.

This conclusion is no longer true when the power consumed during sleep mode $\left(P_{s}\right)$ and the maximum transmit power $\left(P_{\max }\right)$ increase. In particular, 
this conclusion is not true for macrocells and microcells. For these base station types, it is more difficult to find the compromise sought. This case will be studied in a future work.

\section{Acknowledgment}

Part of this work is supported by the project SOGREEN (Smart pOwer Grid for Energy Efficient small cell Networks), which is funded by the French national research agency, under the grant agreement coded: N ANR-14-CE28-0025-02 and by Région Bretagne, France.

\section{References}

1. A. Fehske, G. Fettweis, J. Malmodin, and G. Biczok, "The global footprint of mobile communications: The ecological and economic perspective," Communications Magazine, IEEE, vol. 49, pp. 55-62, August 2011.

2. L. Saker, S.-E. Elayoubi, and T. Chahed, "Minimizing energy consumption via sleep mode in green base station," in Wireless Communications and Networking Conference (WCNC), 2010 IEEE, pp. 1-6, April 2010.

3. M. Naoues, H. Noureddine, Q. Bodinier, H. Zhang, and J. Palicot, "Wifi-based platform for energy saving in wireless networks," in Online Conference on Green Communications (GreenCom), 2014 IEEE, November 2014.

4. EARTH project deliverable 4.2, Green Radio Technologies, January 2012.

5. D. Migliorini, G. Stea, M. Caretti, and D. Sabella, "Power-aware allocation of mbsfn subframes using discontinuous cell transmission in lte systems," in Vehicular Technology Conference (VTC Fall), 2013 IEEE 78th, pp. 1-5, Sept 2013.

6. P. Frenger, P. Moberg, J. Malmodin, Y. Jading, and I. Godor, "Reducing energy consumption in lte with cell dtx," in Vehicular Technology Conference (VTC Spring), 2011 IEEE 73rd, pp. 1-5, May 2011.

7. A. Chatzipapas, S. Alouf, and V. Mancuso, "On the minimization of power consumption in base stations using on/off power amplifiers," in Online Conference on Green Communications (GreenCom), 2011 IEEE, pp. 18-23, Sept 2011.

8. H. Holtkamp, G. Auer, and H. Haas, "On minimizing base station power consumption," in Vehicular Technology Conference (VTC Fall), 2011 IEEE, pp. 1-5, Sept 2011.

9. J. Palicot, H. Zhang, and C. Moy, "On the road towards green radio," The Radio Science Bulletin, pp. 40-56, Dec. 2013.

10. R. Corless, G. Gonnet, D. Hare, D. Jeffrey, and D. Knuth, "On the lambertw function," Advances in Computational Mathematics, vol. 5, no. 1, pp. 329-359, 1996.

11. G. Auer, V. Giannini, C. Desset, I. Godor, P. Skillermark, M. Olsson, M. Imran, D. Sabella, M. Gonzalez, O. Blume, and A. Fehske, "How much energy is needed to run a wireless network?," Wireless Communications, IEEE, vol. 18, pp. 40-49, October 2011.

12. H. Holtkamp, G. Auer, and H. Haas, "Minimal average consumption downlink base station power control strategy," in Personal Indoor and Mobile Radio Communications (PIMRC), 2011 IEEE 22nd International Symposium on, pp. 2430-2434, Sept 2011. 
13. ITU Recommandation, Propagation data and prediction methods for the planning of indoor radiocommunication systems and radio local area networks in the frequency range $300 \mathrm{MHz}$ to $100 \mathrm{GHz}$, July 2015. 